\title{
Article \\ The ‘Oumuamua Encounter: How Modern Cosmology Handled Its First Black Swan
}

\author{
Les Coleman (iD)
}

Citation: Coleman, L. The

'Oumuamua Encounter: How Modern Cosmology Handled Its First Black Swan. Symmetry 2021, 13, 510. https://doi.org/10.3390/sym13030510

Academic Editor: Maxim

Yu. Khlopov

Received: 19 February 2021

Accepted: 17 March 2021

Published: 20 March 2021

Publisher's Note: MDPI stays neutral with regard to jurisdictional claims in published maps and institutional affiliations.

Copyright: (c) 2021 by the author Licensee MDPI, Basel, Switzerland. This article is an open access article distributed under the terms and conditions of the Creative Commons Attribution (CC BY) license (https:// creativecommons.org/licenses/by/ $4.0 /)$
Department of Finance, The University of Melbourne, Parkville, VIC 3010, Australia; les.coleman@unimelb.edu.au; Tel.: +61-38-344-3696

\begin{abstract}
The first macroscopic object observed to have come from outside the solar system slipped back out of sight in early 2018. 1I/2017 U1 'Oumuamua offered a unique opportunity to test understanding of gravity, planetary formation and galactic structure against a true outlier, and astronomical teams from around the globe rushed to study it. Observations lasted several months and generated a tsunami of scientific (and popular) literature. The brief window available to study 'Oumuamua created crisis-like conditions, and this paper makes a comparative study of techniques used by cosmologists against those used by financial economists in qualitatively similar situations where data conflict with the current paradigm. Analyses of 'Oumuamua were marked by adherence to existing paradigms and techniques and by confidence in results from self and others. Some, though, over-reached by turning uncertain findings into graphic, detailed depictions of 'Oumuamua and making unsubstantiated suggestions, including that it was an alien investigator. Using a specific instance to test cosmology's research strategy against approaches used by economics researchers in comparable circumstances is an example of reverse econophysics that highlights the benefits of an extra-disciplinary lens.
\end{abstract}

Keywords: 'Oumuamua; interstellar objects; black swan event; non-gravitational acceleration; interdisciplinary research; reverse econophysics

\section{Introduction}

On 19 October 2017, astronomers in Hawaii sighted what turned out to be an elongated, dark object. Based on its high speed and unusual trajectory, the object was obviously not in solar orbit, and it quickly became accepted as the first body to be observed that had come from outside the solar system. It was named 'Oumuamua, which is Hawaiian for scout or messenger, and remained the subject of intense scrutiny until 2 January 2018 when it became too faint to see, even from the Hubble space telescope [1-4]. 'Oumuamua displayed a variety of puzzling behaviors and proved to be a genuinely seminal encounter, which attracted great interest from physicists and non-specialists across many fields, and laid the foundations for a new discipline that studies material from outside the solar system.

In 2017, scientific expectations for sizeable interstellar objects (ISO) had been clear: they were rare, prospects for identifying one "appear to be bleak" [5], and the most likely candidates were easy to see icy comets or asteroids. Contact with a dark rock prompted upward revision of ISOs' expected spatial density by a factor of several thousand [6]. As a true outlier, 'Oumuamua was modern cosmology's first black swan (Black Swan was a metaphor that London wags used for non-existent phenomena until Dutch navigator Willem de Vlamingh encountered them in 1697 on Perth's aptly named Swan River. Nassim Taleb used this as the theme of his book, The Black Swan: The impact of the highly improbable) and its many unexpected properties provided an opportunity to test astronomical theories [7].

The 'Oumuamua encounter serves as a natural experiment for extra-disciplinary evaluation of modern cosmology's research strategy as set out in leading scientific journals. 
This paper's research objective is to make a comparative study of how researchers in physics and economics disciplines have handled observations that do not conform to the existing paradigm by evaluating physicists' analysis of 'Oumuamua against economists' approach to qualitatively similar situations.

The comparative scrutiny involved study of papers with an eye to their compilation of data and its analysis. At the start of 2021, Discovery database listed 157 peer-reviewed journal articles with 'Oumuamua among their keywords (double the number of two years earlier). About half addressed 'Oumuamua's trajectory and physical properties, and these formed the core sample. Other papers were interpretative and addressed topics such as the origin of 'Oumuamua and the probable distribution of similar objects, or used it as an exemplar in discussion of varied topics such as responses to discovery of similar objects. Each paper was evaluated in light of its implications for the existing cosmological paradigm and the breadth of its conclusions. The intuition is that research publications should not merely showcase knowledge of the current paradigm but use findings to test hypotheses so that existing theory can - as required-be reimagined.

It seems appropriate to evaluate researchers' response to 'Oumuamua in terms of the scientific method, and the following sections, respectively, discuss observation and data collection, and hypotheses derived in relation to 'Oumuamua's features and natural history; then compare cosmologists' study of 'Oumuamua against two qualitatively similar examples from finance, and discuss the relative strengths and weaknesses of the two disciplines' research.

\section{Data Obtained from Cosmologists' Observations}

'Oumuamua was traveling several times faster than the speed of any man-made object, which ruled out close approach and confined research to remote observation across visible and electromagnetic spectra. This section addresses published studies of 'Oumuamua.

Starting with physical features, 'Oumuamua's remained vague. It exhibited chaotic, time-varying behavior [8], including variation by a factor of ten in the brightness of its light curve [9]. This permitted multiple interpretations of its dimensions and shape [10], and the most accurate physical description of 'Oumuamua is that it is 240 [11]-1000 [1] meters long; has an elongated shape consistent with that of a cigar [1] or pancake [12]; is tumbling, with a rotation period between 3 [13] and 55 [12] hours; is moving at between $10 \mathrm{~km} / \mathrm{s}$ [14] and $26 \mathrm{~km} / \mathrm{s}$ [15] relative to the local standard of rest; and is red [1], inhomogeneous, variable or spotted [16], dark or largely neutral [17] in color. Comparison of light curve observations over two months identified brightening that suggests even more elongation [6], and 'Oumuamua's composition was not established. About the only feature that observers agree on is that 'Oumuamua has no detectable emissions of gas or matter $[8,11,18]$, which is confirmed by the lack of any electronic emissions [10], absence of meteorite activity on Earth when it passed in 'Oumuamua's wake that would indicate dust emission [19], and a rate of spin, which is too low for the torque that would result from outgassing [20].

Another physical aspect of 'Oumuamua is its trajectory, which also proved highly uncertain. Positional data showed non-gravitational acceleration (NGA), which is typical of active, comet-like bodies. As a NASA press release (number 18-056 of 28 June 2018) put it, when 'Oumuamua disappeared from view close to Neptune's orbit, it was 40,000 $\mathrm{km}$ further away than if only gravitational force had been acting. NGA was reported as $4.92 \pm 0.16 \times 10^{-6} \mathrm{~m} / \mathrm{s}^{2}$ at a distance of $1 \mathrm{AU}$ from the sun [21]. This radially outward acceleration is about one-thousandth of solar gravitation and was interpreted as resulting from ejection of about $10 \mathrm{~kg}$ of mass each second towards the sun at a velocity of $300 \mathrm{~m} / \mathrm{s}$ [22]. Conversely, a number of studies concluded that 'Oumuamua could not have NGA as large as reported [20,23].

Details of 'Oumuamua were inevitably imprecise because it is an intrinsically faint body, and observations were made from distances in excess of 40 million kilometers, which is equivalent in scale to observing a grain of beach sand from a satellite in Earth 
orbit. 'Oumuamua could not be directly imaged and was too indistinct to measure; because it was already moving away from Earth when sighted, brightness faded rapidly after discovery [24].

Also complicating determination of 'Oumuamua's features is the absence of agreement on the optimum analytical models [25]. Calculations of its dimensions, for instance, assumed that a body stabilizes by rotating around its shortest axis, and so its light curve's periodicity will be set by the ratio of lengths of the two longest axes; and also that the light curve's amplitude is related to an object's size and surface scattering properties. Hence, estimates of 'Oumuamua's dimensions depend on the interpretation of faint photometric imagery and untestable assumptions about its surface properties and motion [24,26].

To summarize, 'Oumuamua's distance, brief transit, small size, and dark appearance brought wide disparity in reports of its dimensions, shape, composition and velocity, which elicited the droll understatement that "these facts . . a are far from definitive" [27].

\section{Hypotheses in Relation to ‘Oumuamua}

After compiling observations of 'Oumuamua, the principal puzzles centered on its natural history, unusual physical features, causes of NGA and its previous trajectory.

Several papers advanced explanations for 'Oumuamua characteristics that are unheard of in the solar system. One proposed [28] that its elongated shape must have been acquired during 'Oumuamua's interstellar journey, with the unstated assumption that no conditions exist anywhere to form anything other than the roughly spherical shapes that characterize the solar system. Another discussed 'Oumuamua's excited rotational state, and argued that-because of the rarity of collisions in space-it must have been acquired long ago in its home planetary system [8], which dismissed the possibility of significant interaction during its interstellar journey.

'Oumuamua's anomalous acceleration away from the sun became linked to explanations for behavior of comet-like objects. Thus, detailed analysis of its light curve concluded that 'Oumuamua was most likely "a thin disc (slab) experiencing moderate torque from outgassing" [25], and the most authoritative survey of its NGA [21] found "comet-like outgassing to be the most physically plausible explanation". The nature of the outgassing, however, was not agreed upon. For instance, it was proposed that 'Oumuamua's NGA and shape could be explained by the presence of hydrogen ice rather than other volatiles [29], but modeling showed such a structure could not survive interstellar radiation [30]. In addition, the required rate of ejection was high relative to its inferred mass [22]. Thus, other alternatives emerged. One paper dismissed any consistency between 'Oumuamua and previously observed transient bodies [18] and proposed it as a thin ( $<1 \mathrm{~mm}$ thick), lowdensity $\left(0.1\right.$ ton $\left./ \mathrm{m}^{3}\right)$ object that was accelerated outwards by solar radiation, which implies a new class of material that could be the product of alien craftsmanship. This conflicted with published estimates [31] of 'Oumuamua's density that exceeded $1.5 \mathrm{ton} / \mathrm{m}^{3}$ and the absence of cohesive or tensile properties consistent with a light sail.

Any interstellar visitor naturally promotes interest in its history, and after 'Oumuamua's trajectory had been described, it should be relatively straightforward to backtrack. An analysis attempted to retrace 'Oumuamua's path to perihelion a month before discovery where it should have been captured by solar observatories, but that proved unsuccessful [32]. Other studies went further back in time, though, to determine 'Oumuamua's original home star or galaxy [33-35]. While a number of candidates were identified, none was conclusive.

\section{Reverse Econophysics}

Econophysics has become a new subdiscipline that introduced insights from physics to explain stylized economic facts. This section looks at lessons for physics in the strategies of economics researchers, using two topics that are qualitatively similar to 'Oumuamua, namely the global financial crisis (GFC) of 2008 and evidence that investors behave irrationally in not following precepts of modern portfolio theory (MPT). 
An issue shared by physics and economics is the Duhem-Quine (DQ), or joint test, problem where interpreting observations must rely on untested theory [36]. The 'Oumuamua joint test problem involves its NGA, which does not match expectations from Newton's law and/or general relativity and is explained by outgassing that is assumed rather than demonstrated. Neither law of gravity has been conclusively proven, and calculating NGA introduces multiple human-based dependencies. Thus, 'Oumuamua's unexpected NGA may be due to shortcomings in theoretical assumptions, mundane errors in observation or analysis, or a genuine anomaly. A similar situation applies to other analyses that are modeldependent or rely on untested assumptions, such as albedo and rotation in calculation of dimensions.

Economics has similar issues, such as assessing investors' rationality against MPT, which is unproven. Its research takes advantage of observations made separately by groups that use a variety of different, sometimes unrelated, techniques, equipment and software; and deliberately marshals disparate research efforts to bring a strong focus on crises and behavioral puzzles. One approach is single topic conferences. When Queen Elizabeth visited the London School of Economics shortly after the GFC and asked, "Why did no one see the crisis coming?" the British Academy responded by holding a specialist seminar around this puzzle [37]. Although there have been conferences on other physics topics such as gravitation, none seems to have been held for 'Oumuamua. A longer-lived focus comes from establishment of centers that promote multidisciplinary evaluation of clearly defined questions. The standalone Santa Fe Institute, for example, selects large problems, such as why some economies outperform and engages researchers from dozens of disciplines [38].

It is common in economics and other social sciences to form subdisciplines to tackle intransigent puzzles. One of the most significant extra-disciplinary initiatives came after the 1960s when psychologists applied their new cognitive models of decision-making to investment and founded the discipline of behavioral economics that brought a Nobel Prize to Daniel Kahneman [39]. As other disciplines' tools and techniques extended with technology, behavioral economists embraced them, such as enlisting neuroscientists and MRIs to study investors' brains while making decisions [40]. Although it has been suggested that 'Oumuamua should prompt a new branch of astronomical research to study interstellar objects [41], progress has been slight.

Economics, psychology and other social sciences make use of data sets involving different geographies, periods and data collection techniques that enable independent development of hypotheses and replication of findings. The potential for insights into 'Oumuamua is indicated by the variety of observations from different teams [12,16,24,26]: many display outliers, short-lived anomalies and patterns that are suggestive of qualitative discrepancies, which might lead to contrasting conclusions if they were analyzed separately as individual datasets. These potential learning opportunities were passed up, though, through the standard practice in cosmology of co-mingling published and shared data; and by scant reports of complex techniques and withholding of proprietary software, e.g., [22]. Even when skill sets were combined, such as through the 'Oumuamua International Space Science Institute (ISSI) Team, the perspective was narrow as no member had a background from other sciences, such as chemistry and geology, much less insights from systems analysts or social scientists.

An issue which economics and other social and biophysical sciences share with physics is that as much as 90 percent of observations do not match the prevailing paradigm. The typical response is to incorporate some form of dark matter, which serves as a placeholder until an explanation emerges. For example, economics relies on an invisible hand to explain how demand and supply automatically reach equilibrium and on psychological biases to explain why investors do not rationally price assets. Puzzles prove resilient when processes are invisible and generate no data and in closed-loop systems with nonlinear dynamics. Complexity theory is a multidisciplinary approach that is making progress [42].

Considering the application of some of these economics research techniques to physics offers the possibility of innovative perspectives. 


\section{Discussion: Strengths and Weaknesses in 'Oumuamua Research}

An impressive aspect of research into 'Oumuamua was the speed with which information was published. The Astrophysical Journal Letters took the lead with an initial paper published on 30 November 2017 and over 30 since. Most disciplines measure time to publication in months or years, whereas AJL took days, and other journals were comparably efficient. Invariably, papers were of high quality, evidencing good preparation and tight editing, and offered actionable insights from cosmologists' research. Importantly, most papers avoided jargon and assumed modest pre-knowledge, so they were readily accessible to interested non-cosmologists.

The most striking feature of the hundreds of articles about 'Oumuamua was confidence in conclusions that largely relied on observations in visible light, which produced no more evidence than is contained in a few pixels [17,43]. Despite that, scientists provided authoritative descriptions right down to commissioning artists' impressions showing the object in extraordinary detail [44]. As another example, 'Oumuamua's track could not be unwound more than a few days before discovery; there was no information about its inbound trajectory and no agreement on the magnitude of any anomalous outbound acceleration. That should rule out credible suggestions about its past or future journey, but multiple authors did not agree and canvassed its origins and destination.

A second striking feature was researchers' self-restraint in respecting colleagues' contributions and cleaving to established paradigms. Unraveling the enigma of 'Oumuamua demanded multiple skills in observation and analysis, which forced researchers to rely on fellow scientists' conclusions. These interdependencies were marked by collegiality, with a refusal to contemplate turf wars. Few authors questioned material they relied on, and most were at pains to point out consistency of their results with those of colleagues. Given difficulties in observation, it was surprising to see only a few dissenting conclusions drawn from shared data [examples include $[20,23,25,27,30,45]$. Few researchers challenged the reasonableness of even the most improbable findings.

There was matching self-restraint in interpreting the obvious conclusion from photometric comparisons that 'Oumuamua was extreme relative to comets and asteroids [6,46]. Researchers were unwilling to tinker with the existing paradigm, which implied that the solar system contains a reliable sample of all objects in the universe. With no additional information expected to be found from an interstellar data point, 'Oumuamua was not studied de novo, and its features were given conventional explanations. By contrast, economics tends to look at data in isolation and develop hypotheses.

Examples of socializing 'Oumuamua's surprises can be seen in analyses of its trajectory, which is intuitively its most obvious and easily determined feature. It also offers out-ofsample tests, which are the best demonstration of how well any phenomenon is understood. This was demonstrated with a second ISO 2I/Borisov, which was similar to solar system comets [47], and-although detected in August 2019 when inbound about 3 AU from the sun - was successfully identified in earlier images dating back to late 2018 when it was 7.8 AU from the sun [48]. By contrast, there was no success in a similar attempt to backtrack 'Oumuamua beyond a few days, so it does not seem reasonable to place any credibility on conclusions regarding its trajectory.

Another example of doubtful conclusions relates to NGA, which is commonly observed with similar objects, and was reported for 'Oumuamua with $30 \sigma$ significance [21]. Its velocity, though, was far above that of any solar system body and comparable to that of nearby stars $[1,26]$, and its NGA is an order of magnitude above the median and in the top decile [49]. In addition, NGA confidence dropped to as low as $2.5 \sigma$ using subsets of the data, and other analyses did not detect any non-gravitational forces [34]. Given puzzles in theoretical understanding of gravity and previous significant errors in similar calculations, it would have been useful to fully support findings. Despite this, the paper reporting NGA provides some observations and pointers to freely available software, but code is withheld as proprietary, and numerical findings are only briefly reported [50]. The result is that 
NGA essentially emerges from a black-box, and readers are left with no alternative but to accept the calculation.

Out-of-sample tests show that 'Oumuamua's trajectory was not accurately determined, and the claimed level of significance for its NGA does not seem reasonable. Although 'Oumuamua's striking NGA could result from repulsion by the sun (such as solar radiation), attraction by a distant object (which is consistent with its hyperbolic trajectory), an inherent property, or provide evidence of inapplicability of Newtonian gravity, it was attributed without any evidentiary support to outgassing [20]. A challenge suggesting a constant acceleration vector was dismissed as not matching data any better than outgassing [21].

A further challenge to conventional explanations of 'Oumuamua's natural history was the suggestion that it was a product of alien artifice. Even though there was zero supporting evidence, the explanation faced little argument, perhaps because 'Oumuamua was sighted on the day after the 50th anniversary of the first telecast of one of Star Trek's most popular episodes in which the similarly shaped doomsday machine nearly ended voyages of starship Enterprise [51]. The attribution to aliens gained some currency but was studied in conventional terms within existing paradigms (an exception is reference [52]). For instance, communications between aliens and 'Oumuamua were ruled out based on reviews of emissions across standard electromagnetic wavelengths [53,54]. If an alien civilization were using such an exotic tool for interstellar exploration, surely it would communicate through something more sophisticated than our 5G wireless network. A true assessment would have taken 'Oumuamua's proximity as an unprecedented opportunity to detect even weak alien signals (and evaluate other exotic aspects), which-assuming that alien instruments work best at close range-would peak during or soon after reaching target-rich settings. Search for alien signals would have looked to granular geo- and astrophysical data from neutrino detectors and similar, where anomalies common to days when 'Oumuamua passed near the sun and planets would be a powerful tale. (Reuters reports contain nothing "weird" on 8 October 2017 when Oumuamua passed closest to Earth, and UFO sightings were only a little above-average during 'Oumuamua's transit: see National UFO Reporting Center data at www.nuforc.org). Another avenue to explore is any significance in what we know as fact, such as 'Oumuamua's velocity, distances of its Earth and solar flybys, and its next destination. Perhaps its overactive light curve holds a clue.

\section{Conclusions}

Science has a global constituency, so 'Oumuamua became a media highlight. It is impractical for the public, which funds such work, to know how much reliance to place on scientific research, but 'Oumuamua enables some evaluation. In reality, information about Oumuamua is sufficiently vague to accommodate multiple hypotheses. Where this counseled caution, it all too often promoted speculation promoted as fact. In addition, although 'Oumuamua's exceptions provided a unique opportunity to evaluate existing assumptions, little effort was made through comparison of different teams' observations, sharing techniques in the spirit of Open Science, and bringing insights from experts in other physical and social sciences. Anomalies were simply shoehorned into the existing paradigm, which is a well-worn path for physicists, who invent something unknown to solve a puzzle without upsetting the paradigm and have seen ether, dark matter, dark energy and alien civilizations [55] paper over other disturbing theoretical cracks.

Although a sample of one, 'Oumuamua offered an exciting learning opportunity that could catalyze reimagining of cosmological phenomena. However, to protect troubled paradigms scientists simply airbrushed out its puzzles through patchwork modifications that were as improbable as alien artifice.

A number of ideas are suggested by a comparative analysis of physics and economics research. Planning for studies of future interstellar objects may provide for greater cooperation between teams to maximize the breadth of observations without duplication; agree and publish bespoke tools and software to analyze NGA, rotation, emissions and 
other puzzles, which would quickly identify anomalous findings and facilitate replication; access observations from non-cosmological instruments; and bring geoscientists, systems analysts and social scientists onto study teams to catalyze ideas. A multi-day conference on 'Oumuamua could revisit data without prejudgments; build on its puzzles and surprises to develop specific research questions for future studies, and formalize a new discipline to study interstellar objects. Finally, 'Oumuamua's inexplicable NGA adds to the long list of well-documented gravitation puzzles in the solar system, e.g., [56] that justify intense scrutiny of Newton's Law.

In closing, implications of the discussion above are clear: as a true black swan, 'Oumuamua was an outlier object that was quite different from expectations of interstellar bodies. It is one of the most elongated objects observed, is tumbling, not spinning, has remarkable photometric variability, and is traveling fast in a unique orbit. Many, perhaps most, of 'Oumuamua's parameters lie well into the tails of near-Earth samples [4], and it has numerous inexplicable features. As observations of 'Oumuamua grew, its physics, chemistry and behavior became less and less like anything previously encountered. If it is representative of a significant portion of interstellar objects, then assumptions based on data compiled on Earth may not encompass distant settings, and the existing paradigm would not be relevant on galactic and cosmic scales. That, of course, describes the current situation.

Funding: This research did not receive any specific grant from funding agencies in the public, commercial, or not-for-profit sectors.

Institutional Review Board Statement: Not applicable.

Informed Consent Statement: Not applicable.

Data Availability Statement: No new data were created or analyzed in this study. Data sharing is not applicable to this article.

Conflicts of Interest: The author declares that he has no known competing financial interests or personal relationships that could have appeared to influence the work reported in this paper. There are no co-authors to the article, and I confirm sole authorship. There is no figure or text in the article that has been taken from another paper without attribution.

\section{References}

1. Meech, K.J.; Weryk, R.; Micheli, M.; Kleyna, J.T.; Hainaut, O.R.; Jedicke, R.; Wainscoat, R.J.; Chambers, K.C.; Keane, J.V.; Petric, A. A brief visit from a red and extremely elongated interstellar asteroid. Nature 2017, 552, 378-381. [CrossRef]

2. Hainaut, O.R.; Meech, K.J.; Micheli, M.; Belton, M.S. Rendezvous withOumuamua. Messenger 2018, $173,13-16$.

3. Seligman, D.; Laughlin, G. The feasibility and benefits of in situ exploration of 'Oumuamua-like objects. Astron. J. $2018,155,217$. [CrossRef]

4. Bannister, M.T.; Bhandare, A.; Dybczyński, P.A.; Fitzsimmons, A.; Guilbert-Lepoutre, A.; Jedicke, R.; Knight, M.M.; Meech, K.J.; McNeill, A.; Pfalzner, S.; et al. The natural history of 'Oumuamua. Nat. Astron. 2019, 3, 594-602. [CrossRef]

5. Engelhardt, T.; Jedicke, R.; Vereš, P.; Fitzsimmons, A.; Denneau, L.; Beshore, E.; Meinke, B. An observational upper limit on the interstellar number density of asteroids and comets. Astron. J. 2017, 153, 133. [CrossRef]

6. Siraj, A.; Loeb, A. Identifying interstellar objects trapped in the solar system through their orbital parameters. Astrophys. J. Lett. 2019, 872, L10. [CrossRef]

7. Klesman, A. 'Oumuamua: Our first interstellar visitor. Astronomy 2020, 10.

8. Drahus, M.; Guzik, P.; Waniak, W.; Handzlik, B.; Kurowski, S.; Xu, S. Tumbling motion of 1I/‘Oumuamua and its implications for the body's distant past. Nat. Astron. 2018, 2, 407. [CrossRef]

9. Vazan, A.; Sari, R.e. On the aspect ratio of 'Oumuamua: Less elongated shape for irregular surface properties. Mon. Not. R. Astron. Soc. 2020, 493, 1546-1552. [CrossRef]

10. Fitzsimmons, A.; Snodgrass, C.; Rozitis, B.; Yang, B.; Hyland, M.; Seccull, T.; Bannister, M.T.; Fraser, W.C.; Jedicke, R.; Lacerda, P. Spectroscopy and thermal modelling of the first interstellar object 1I/2017 U1 'Oumuamua. Nat. Astron. 2018, 2, $133-137$. [CrossRef]

11. Trilling, D.E.; Mommert, M.; Hora, J.L.; Farnocchia, D.; Chodas, P.; Giorgini, J.; Smith, H.A.; Carey, S.; Lisse, C.M.; Werner, M. Spitzer observations of interstellar object 1I/‘Oumuamua. Astron. J. 2018, 156, 261. [CrossRef]

12. Belton, M.J.; Hainaut, O.R.; Meech, K.J.; Mueller, B.E.; Kleyna, J.T.; Weaver, H.A.; Buie, M.W.; Drahus, M.; Guzik, P.; Wainscoat, R.J. The excited spin state of 1I/2017 U1 'Oumuamua. Astrophys. J. Lett. 2018, 856, L21. [CrossRef] 
13. Knight, M.M.; Protopapa, S.; Kelley, M.S.; Farnham, T.L.; Bauer, J.M.; Bodewits, D.; Feaga, L.M.; Sunshine, J.M. On the rotation period and shape of the hyperbolic asteroid 1I/‘Oumuamua (2017 U1) from its lightcurve. Astrophys. J. Lett. 2017, 851, L31. [CrossRef]

14. Mamajek, E. Kinematics of the interstellar vagabond 1I/‘Oumuamua (A/2017 U1). Res. Notes Aas 2017, 1, 21. [CrossRef]

15. Feng, F.; Jones, H.R. 'Oumuamua as a Messenger from the Local Association. Astrophys. J. Lett. 2018, 852, L27. [CrossRef]

16. Fraser, W.C.; Pravec, P.; Fitzsimmons, A.; Lacerda, P.; Bannister, M.T.; Snodgrass, C.; Smolić, I. The tumbling rotational state of 1I/‘Oumuamua. Nat. Astron. 2018, 2, 383. [CrossRef]

17. Bannister, M.T.; Schwamb, M.E.; Fraser, W.C.; Marsset, M.; Fitzsimmons, A.; Benecchi, S.D.; Lacerda, P.; Pike, R.E.; Kavelaars, J.; Smith, A.B. Col-OSSOS: Colors of the interstellar planetesimal 1I/‘Oumuamua. Astrophys. J. Lett. 2017, 851, L38. [CrossRef]

18. Bialy, S.; Loeb, A. Could solar radiation pressure explain ‘Oumuamua's peculiar acceleration? Astrophys. J. Lett. 2018, 868, L1-L5. [CrossRef]

19. Rafikov, R.R. Spin Evolution and Cometary Interpretation of the Interstellar Minor Object 1I/2017 ‘Oumuamua. Astrophys. J. Lett. 2018, 867, L17. [CrossRef]

20. Micheli, M.; Farnocchia, D.; Meech, K.J.; Buie, M.W.; Olivier, R.H.; Prialnik, D.; Norbert, S. Non-gravitational acceleration in the trajectory of 1I/2017 U1 (‘Oumuamua). Nature 2018, 559, 223-226. [CrossRef]

21. Seligman, D.; Laughlin, G.; Batygin, K. On the anomalous acceleration of 1I/2017 U1 ‘Oumuamua. Astrophys. J. Lett. 2019, 876, L26. [CrossRef]

22. Ye, Q.-Z.; Zhang, Q.; Kelley, M.S.; Brown, P.G. 1I/2017 U1 (‘Oumuamua) is hot: Imaging, spectroscopy, and search of meteor activity. Astrophys. J. Lett. 2017, 851, L5. [CrossRef]

23. Katz, J. Evidence against non-gravitational acceleration of 1I/2017 U1 ‘Oumuamua. Astrophys. Space Sci. 2019, 364, 51. [CrossRef]

24. Bolin, B.T.; Weaver, H.A.; Fernandez, Y.R.; Lisse, C.M.; Huppenkothen, D.; Jones, R.L.; Jurić, M.; Moeyens, J.; Schambeau, C.A.; Slater, C.T. APO time-resolved color photometry of highly elongated interstellar object 1I/‘Oumuamua. Astrophys. J. Lett. 2017, 852, L2. [CrossRef]

25. Mashchenko, S. Modelling the light curve of 'Oumuamua: Evidence for torque and disc-like shape. Mon. Not. R. Astron. Soc. 2019, 489, 3003-3021. [CrossRef]

26. Gaidos, E. What and whence 1I/‘Oumuamua: A contact binary from the debris of a young planetary system? Mon. Not. R. Astron. Soc. 2018, 477, 5692-5699. [CrossRef]

27. Forbes, J.C.; Loeb, A. Turning up the Heat on 'Oumuamua. Astrophys. J. Lett. 2019, 875, L23. [CrossRef]

28. Vavilov, D.E.; Medvedev, Y.D. Dust bombardment can explain the extremely elongated shape of 1I/'Oumuamua and the lack of interstellar objects. Mon. Not. R. Astron. Soc. Lett. 2019, 484, L75-L78. [CrossRef]

29. Seligman, D.; Laughlin, G. Evidence that 1I/2017 U1 ('Oumuamua) was composed of molecular hydrogen ice. Astrophys. J. Lett. 2020, 896, L8. [CrossRef]

30. Hoang, T.; Loeb, A. Destruction of molecular hydrogen ice and implications for 1I/2017 U1 ('Oumuamua). Astrophys. J. Lett. 2020, 899, L23. [CrossRef]

31. McNeill, A.; Trilling, D.E.; Mommert, M. Constraints on the density and internal strength of 1I/‘Oumuamua. Astrophys. J. Lett. 2018, 857, L1. [CrossRef]

32. Hui, M.-T.; Knight, M.M. New insights into interstellar object 1I/2017 U1 (‘Oumuamua) from SOHO/STEREO nondetections. Astron. J. 2019, 158, 256. [CrossRef]

33. Bailer-Jones, C.A.; Farnocchia, D.; Meech, K.J.; Brasser, R.; Micheli, M.; Chakrabarti, S.; Buie, M.W.; Hainaut, O.R. Plausible home stars of the interstellar object 'Oumuamua found in Gaia DR2. Astron. J. 2018, 156, 205. [CrossRef]

34. Dybczynski, P.A.; Królikowska, M.; Kim, J.; Lee, S.; Hodgson, J.; Algaba, J.; Zhao, G.; Kino, M.; Byun, D.; Kang, S. Investigating the dynamical history of the interstellar object 'Oumuamua. Astron. Astrophys. 2018, 610, L11. [CrossRef]

35. Portegies Zwart, S.; Torres, S.; Pelupessy, I.; Bédorf, J.; Cai, M.X. The origin of interstellar asteroidal objects like 1I/2017 U1 ‘Oumuamua. Mon. Not. R. Astron. Soc. Lett. 2018, 479, L17-L22. [CrossRef]

36. Harding, S. Can Theories Be Refuted?: Essays on the Duhem-Quine thesis; Springer Science \& Business Media: Berlin, Germany, 1975; Volume 81.

37. Besley, T.; Hennessy, P. The Global Financial Crisis-Why didn't anybody notice? Br. Acad. Rev. 2009, 14, 8-11.

38. Buyalskaya, A.; Gallo, M.; Camerer, C.F. The golden age of social science. Proc. Natl. Acad. Sci. USA 2021, 118, e2002923118. [CrossRef]

39. Kahneman, D. Thinking, Fast and Slow; Farra, Straus and Giroux: New York, NY, USA, 2011.

40. Srivastava, M.; Sharma, G.D.; Srivastava, A.K.; Kumaran, S.S. What's in the brain for us: A systematic literature review of neuroeconomics and neurofinance. Qual. Res. Financ. Mark. 2020. [CrossRef]

41. Siraj, A.; Loeb, A. Probing Extrasolar Planetary Systems with Interstellar Meteors. arXiv 2019, arXiv:1906.03270.

42. Hidalgo, C.A. Economic complexity theory and applications. Nat. Rev. Phys. 2021, 1-22.

43. Trilling, D.E.; Robinson, T.; Roegge, A.; Chandler, C.O.; Smith, N.; Loeffler, M.; Trujillo, C.; Navarro-Meza, S.; Glaspie, L.M. Implications for planetary system formation from interstellar object 1I/2017 U1 (‘Oumuamua). Astrophys. J. Lett. 2017, 850 , L38. [CrossRef]

44. Laughlin, G. ‘Oumuamua's dramatic visit. Sky Telesc. 2018, 136, 20-27.

45. Wilhelm, K.; Dwivedi, B.N. Anomalous Sun Flyby of 1I/2017 U1 (Oumuamua). Galaxies 2020, 8, 83. [CrossRef] 
46. Jewitt, D.; Luu, J.; Rajagopal, J.; Kotulla, R.; Ridgway, S.; Liu, W.; Augusteijn, T. Interstellar interloper 1I/2017 U1: Observations from the NOT and WIYN telescopes. Astrophys. J. Lett. 2017, 850, L36. [CrossRef]

47. Hui, M.-T.; Ye, Q.-Z.; Föhring, D.; Hung, D.; Tholen, D.J. Physical Characterisation of Interstellar Comet 2I/2019 Q4 (Borisov). arXiv 2020, arXiv:2003.14064.

48. Ye, Q.; Kelley, M.S.; Bolin, B.T.; Bodewits, D.; Farnocchia, D.; Masci, F.J.; Meech, K.J.; Micheli, M.; Weryk, R.; Bellm, E.C. Pre-discovery activity of new interstellar comet 2I/Borisov beyond 5 AU. Astron. J. 2020, 159, 77. [CrossRef]

49. Rafikov, R.R. Non-gravitational forces and spin evolution of comets. arXiv 2018, arXiv:1809.05133.

50. Mottola, S.N.; Attree, L.; Jorda, H.U.; Keller, R.; Kokotanekova, D. Marshall and Y. Skorov. Nongravitational Effects of Cometary Activity. Space Sci. Rev. 2020, 216, 1-20. [CrossRef]

51. Kahn, M. My Favorite Classic Star Trek Episode. Smithson. Mag. 2020.

52. Gertz, J. Oumuamua and Scout ET Probes. arXiv 2019, arXiv:1904.04914.

53. Harp, G.; Richards, J.; Jenniskens, P.; Shostak, S.; Tarter, J.C. Radio SETI observations of the interstellar object 'Oumuamua. Acta Astronaut. 2019, 155, 51-54. [CrossRef]

54. Tingay, S.; Kaplan, D.; Lenc, E.; Croft, S.; McKinley, B.; Beardsley, A.; Crosse, B.; Emrich, D.; Franzen, T.; Gaensler, B. A serendipitous MWA search for narrowband signals from 'Oumuamua. Astrophys. J. 2018, 857, 11. [CrossRef]

55. Wright, J.T.; Cartier, K.M.; Zhao, M.; Jontof-Hutter, D.; Ford, E.B. The $\hat{G}$ search for extraterrestrial civilizations with large energy supplies. Astrophys. J. 2015, 816, 17. [CrossRef]

56. Iorio, L. Gravitational anomalies in the solar system? Int. J. Mod. Phys. D 2015, 24, 1530015. [CrossRef] 